\title{
An adult onset hexosaminidase A deficiency syndrome with sensory neuropathy and internuclear ophthalmoplegia
}

\author{
D Barnes, V P Misra, E P Young, P K Thomas, A E Harding
}

\begin{abstract}
A 42 year old man presented with a slowly progressive gait disturbance, generalised weakness, dysarthria, clumsiness and tremor of his hands, and involuntary jerks. Hexosaminidase A activity in plasma, leucocytes and fibroblasts was considerably reduced, establishing the diagnosis of $\mathbf{G M}_{2}$ gangliosidosis. Clinical examination showed two previously unreported features, a clinically evident sensory neuropathy and internuclear ophthalmoplegia.
\end{abstract}

Chronic $\mathrm{GM}_{2}$ gangliosidosis due to hexosaminidase A deficiency shows considerable clinical heterogeneity, and increasing awareness of this disorder in adults has led to the recognition that it may mimic a wide variety of neurodegenerative disorders. ${ }^{1}$ It is inherited as an autosomal recessive trait, and predominant manifestations in adult onset cases include progressive ataxia, spasticity, neurogenic weakness, dystonia, dementia, seizures and psychiatric disorders. ${ }^{2-7}$ The clinical features, however, show no clear correlation with any of the enzymatic profiles that have so far been identified. ${ }^{6}$ Hexosaminidase A deficiency (including Tay-Sachs disease) arises from a mutation of the alpha subunit of the enzyme, leading to the so-called $B$ variant of the disease. Also identified are the $A B$ variant, caused by a deficiency of the activator protein required for hexosaminidase activity, and the $B 1$ variant in which hexosaminidase $A$ shows an abnormal substrate specificity. The O variant (Sandhoff's disease) arises when a beta subunit mutation causes deficiency of both hexosaminidase $\mathrm{A}$ and $\mathrm{B}$.

Our patient with an adult onset hexosaminidase A deficiency syndrome showed unusual neurological features.

\section{Case report}

This 42 year old man presented with progressive limb weakness and unsteadiness which had necessitated his retirement from crane driving 18 months previously. Since adolescence, running had been difficult and his hands had been tremulous. During his twenties and thirties he had noticed slowly progressive weakness of his legs and clumsiness of gait. After the age of 40 years, he had noticed a marked deterioration in his walking, weakness of his arms, and a jerkiness of movement including breathing. His speech had become difficult to understand and unpredictable in volume. There was no other neurological history, and he did not drink alcohol. His family history was unremarkable, and there was no parental consanguinity or Jewish ancestry.

On examination, cognitive function was normal. He had a slurring, at times explosive, dysarthria. Slow phase eye movements were jerky in all directions, and conjugate upgaze showed a $20-30 \%$ limitation improved by the doll's head manoeuvre. In addition there was a left internuclear ophthalmoplegia (INO) with abducting nystagmus. There was generalised muscle wasting with corresponding weakness in the limbs, more marked proximally. The trunk muscles were also weak. All tendon reflexes were brisk and the plantar responses were flexor. He showed upper limb ataxia, but coordination was difficult to assess in his legs due to weakness. There was a coarse, irregular postural tremor of the hands which increased on action, and a smaller amplitude tremor of the head and neck muscles. Occasional myoclonic jerks were seen in the perioral muscles and both arms. Expiration was frequently interrupted by jerking, probably due to diaphragmatic myoclonus. Pinprick appreciation was absent in the feet and diminished to both knees, and proprioception was impaired to the ankles. Standing from a chair was extremely difficult; his gait was unsteady and rolling and he was unable to walk heel to toe.

The following investigations were normal or negative: routine haematology and biochemistry (including blood sugar and liver function), acanthocyte screen, syphilis serology, vitamin $B_{12}$ and folate, creatine kinase, vitamin $\mathrm{E}$, lipid and protein electrophoresis, chest radiography, and cerebrospinal fluid analysis, including electrophoresis. With the exception of hexosaminidase, lysosomal enzyme activities were normal.

Plasma, leucocyte and fibroblast hexosaminidase activity was assayed using two synthetic substrates: 1) 4-methylumbelliferyl- 
2 - acetamido - 2 - deoxy- $\beta$-D-glucopyranoside, which measures both hexosaminidase $A$ and B; 2) The sulphated substrate 4-methylumbelliferyl-2-acetamido-2-deoxy-6-sulpho$\beta$-D-glycopyranoside which is specific for hexosaminidase $A$. Using the first substrate, the total $\beta$-hexosaminidase activity was within the normal range, but the heat labile hexosaminidase (predominantly A) was reduced to $4 \%$ (normal: $49-70$ ) of the total in leucocytes, $8 \%$ (normal: $52-78$ ) in plasma, and 10\% (normal: 43-62) in cultured fibroblasts. With the sulphated substrate the following profound deficiencies of hexosaminidase A were found: leucocytes $-14 \mathrm{nmol} / \mathrm{mg}$ protein/h (normal: 162-469); plasma-8 $\mathrm{nmol} / \mathrm{ml} / \mathrm{h}$ (92-326); and fibroblasts-60 $\mathrm{nmol} / \mathrm{mg}$ protein/h (428-1120).

Motor nerve conduction studies were normal. The superficial peroneal and saphenous sensory action potentials (SAPs) were absent, and the sural SAP was $3 \mu \mathrm{V}$ in amplitude. The median, ulnar and radial SAP amplitudes were 12,4 , and $28 \mu \mathrm{V}$ respectively. Electromyography showed widespread chronic partial denervation in the upper and lower limbs. Visual and brainstem auditory evoked potentials were normal, but somatosensory potentials were absent with tibial nerve stimulation. Electronystagmography confirmed the presence of a left INO. Magnetic resonance imaging showed marked widening of the cerebellar sulci and atrophy of the brainstem. Formal psychometry suggested that his current dull average level of function was a little below his premorbid performance.

A right sural nerve fascicular biopsy was performed. There was a moderate reduction in the myelinated nerve fibre population, with a fibre density of $4442 \mathrm{~mm}^{-2}$ (normal range $7500-10000 \mathrm{~mm}^{-28}$ ), despite the presence of regenerative clusters. The proportion of large diameter fibres over $7 \mu \mathrm{m}$ was reduced to $19 \%$, compared with $37.5 \%$ in age-matched control nerve. The density of unmyelinated axons was slightly reduced at $24379 \mathrm{~mm}^{-2}$ (normal range $30000-40000 \mathrm{~mm}^{-28}$ ) but they had a normal unimodal size distribution. Moderately numerous stacks of flattened Schwann cell processes unassociated with axons were present, indicating degeneration of unmyelinated axons. No hypertrophic changes were seen, and there were no abnormal inclusions either in Schwann cells or axons. The appearances were those of a chronic neuropathy with predominant axonal degeneration and regeneration, but with no specific features. A skin biopsy, showing a number of small nerve bundles, was normal.

\section{Discussion}

Most of the features of this patient's clinical syndrome were typical of chronic $\mathrm{GM}_{2}$ gangliosidosis. ${ }^{67}$ Specifically, ataxia, neurogenic weakness, intellectual impairment, ocular dysmetria, dysarthria, and myoclonus are all well described, but two features observed here have not previously been reported in this disease, namely a sensory neuropathy and INO.

Although our patient did not have sensory symptoms, impaired pinprick appreciation and proprioception in the legs suggested the presence of a neuropathy which was confirmed by nerve conduction studies and a sural nerve biopsy. Appropriate investigations revealed no alternative aetiology. In previously reported cases of this disorder the sensory examination has been consistently normal, and although Mitsumoto et $\mathrm{al}^{9}$ diagnosed a motor-sensory neuropathy in one of their patients on the basis of small lower limb sensory nerve action potentials (and an axonopathic nerve biopsy), sensory electrophysiology is usually also normal. Thus while clinical sensory abnormalities are very uncommon in chronic $\mathrm{GM}_{2}$ gangliosidosis, they are not inconsistent with the diagnosis.

The second unusual feature in our patient was an INO. Some abnormality of ocular motility has been noted in the majority of patients with hexosaminidase A deficiency reported in the literature. Detailed examination of eye movements may therefore provide evidence of a multisystem disorder in a patient suspected of having amyotrophic lateral sclerosis or spinal muscular atrophy. Various ocular motor disorders have been described in hexosaminidase A deficiency, most commonly those compatible with degeneration of the cerebellum or its connections. Slow or hypometric horizontal and vertical saccades, horizontal nystagmus, restricted horizontal and vertical pursuit, head thrusting reminiscent of ocular "apraxia"6 and supranuclear ophthalmoplegia ${ }^{2}$ have all been reported. To this list may be added INO. In our patient this was unilateral, and although the precise mechanism is not known, it was probably related to extension of the brainstem degenerative process, evident on MRI, to involve the left medial longitudinal fasciculus.

In conclusion, the features of our patient add to the wide range of clinical manifestations seen in chronic $\mathrm{GM}_{2}$ gangliosidosis. This disease should be considered in any undiagnosed multisystem neurodegenerative syndrome, as its identification may have important prognostic and genetic implications for patients and their families.

We thank Dr Philip Kennedy for referring the patient, and Dr R H M King for performing the electron microscopy.

1 Johnson WG. The clinical spectrum of hexosaminidase deficiency diseases. Neurology 1981;31:1453-56.

2 Harding AE, Young EP, Schon F. Adult onset supranuclear ophthalmoplegia, cerebellar ataxia, and neurogenic proximal muscle weakness in a brother and sister: agenic proximalmuscle weakness in a brother and sister: another Neurosurg Psychiatry 1987;50:687-90.

3 Gudesblatt M, Ludman MD, Cohen JA, et al. Hexosaminidase A activity and amyotrophic lateral sclerosis. Muscle Nerve 1988;11:227-30.
Muminidase A activity and amyou

4 Karni A, Navon R, Sadeh M. Hexosaminidase A deficiency manifesting as spinal muscular atrophy of late onset. $A n n$ Neurol 1988;24:451-53.

5 Hardie RJ, Young EP, Morgan-Hughes JA. Hexosaminidase A deficiency presenting as juvenile progressive dystonia. J Neurol Neurosurg Psychiatry 1988;51:446.

6 Specola N, Vanier MT, Goutieres F, Mikol J, Aicardi J. The juvenile and chronic forms of $\mathbf{G M}_{2}$ gangliosidosis. Neurology 1990;40:145-50.

7 Navon R, Argov Z, Frisch A. Hexosaminidase A deficiency in adults. Am J Med Genet 1986;24:179-96.

8 Jacobs JM, Love S. Qualitative and quantitative morphometry of human sural nerve at different ages. Brain 1985;108:897-924.

9 Mitsumoto H, Sliman RJ, Schafer IA, et al. Motor neuron disease and adult hexosaminidase $A$ deficiency in two families: evidence for multisystem degeneration. Ann Neurol 1985;17:378-85. 